\title{
Experiences and perspectives on the GIST patient journey
}

This article was published in the following Dove Press journal:

Patient Preference and Adherence

29 March 2012

Number of times this article has been viewed

\section{Nancy Macdonald' \\ Ari Shapiro' \\ Christina Bender ${ }^{2}$ \\ Marc Paolantonio ${ }^{2}$ \\ John Coombs ${ }^{2}$}

'Flince Research + Design, New York, NY, ${ }^{2}$ Novartis Pharmaceuticals,

East Hanover, NJ, USA
Correspondence: Nancy Macdonald Flince Research + Design, 249 Smith Street, \#190, Brooklyn, NY I I23I, USA Tel + I 6464962379

Fax + I 6462191578

Email nmacdonald@flinceresearch.com
Purpose: The tyrosine kinase inhibitor (TKI) imatinib has improved outcomes for patients with unresectable or metastatic gastrointestinal stromal tumors (GIST), and for patients receiving adjuvant therapy following GIST resection. This qualitative study explored the experiences and emotions of patients through GIST diagnosis, treatment initiation, disease control, and in some patients, loss of response and therapy switch.

Patients and methods: Ethnographic investigations were conducted, including semistructured qualitative interviews of patients with resected or metastatic/unresectable GIST and their caregivers, from Canada $(n=15)$; the United States $(n=10)$; and Brazil, France, Germany, Russia, and Spain ( $n=5$ each). Some interviewees also kept 7-day photo journals. Responses were qualitatively analyzed to identify gaps and unmet needs where communication about disease, treatments, and adherence could be effective.

Results: Patients shared common experiences during each stage of disease management (crisis, hope, adaptation, new normal, and uncertainty). Patients felt a sense of crisis during diagnosis, followed by hope upon TKI therapy initiation. Over time, they came to adapt to their new lives (new normal) with cancer. With each follow-up, patients confronted the uncertainty of becoming TKI resistant and the possible need to switch therapy. During uncertainty many patients sought new information regarding GIST. Cases of disease progression and drug switching caused patients to revert to crisis and restart their emotional journey. Patients with primary or unresectable/metastatic GIST shared similar journeys, especially regarding uncertainty, although differences in the scope and timing of phases were observed. Strategies patients used to remain adherent included obtaining family support, setting reminder mechanisms, taking medicine at routine times, and storing medicine in prominent places.

Conclusions: Physicians and support staff can manage patient expectations and encourage adherence to therapy, which may facilitate optimal patient outcomes. Patient education about current GIST developments and adherence across all phases of the patient journey are of benefit. Keywords: Gastrointestinal stromal tumors, medication adherence, compliance, imatinib, qualitative study, ethnographic investigation

\section{Introduction}

Gastrointestinal stromal tumors (GIST) are the most common mesenchymal tumors of the gastrointestinal tract. ${ }^{1}$ GIST most commonly occur in the stomach $(60 \%-70 \%)$ or small intestine $(20 \%-25 \%)$ at a rate of 20 cases per million persons per year. ${ }^{2,3}$ The introduction of the tyrosine kinase inhibitor (TKI) imatinib (Glivec ${ }^{\circledR} /$ Gleevec $^{\circledR}$, Novartis AG, Basel, Switzerland) has improved the treatment of $\mathrm{KIT}^{+}$GIST. Clinical results have shown improvements in response rates and progression-free survival for patients with unresectable and/or metastatic $\mathrm{KIT}^{+}$GIST treated with imatinib at doses between 
$400 \mathrm{mg} /$ day and $800 \mathrm{mg} /$ day. ${ }^{4,5}$ Interruption of imatinib after 1,3 , or 5 years has been associated with increased risk of relapse, even in patients who achieve complete response, suggesting that continuous therapy may be necessary until disease progression or intolerance occurs. ${ }^{6,7}$ As such, adherence to self-administered imatinib therapy is an essential component of the daily routine of these patients, and adherence evaluation is a key component of disease management for health care providers (HCPs).

The clinical benefit of imatinib as adjuvant therapy, given for 3 years after resection in patients with $\mathrm{KIT}^{+}$GIST, has been validated in the randomized Phase III Scandinavian Sarcoma Group (SSG)/Sarcoma Group of the Arbeitsgemeinschaft Internistische Onkologie (AIO; SSGXVIII/AIO) trial. ${ }^{8}$ Although surgery is considered the only curative treatment for primary resectable GIST, even after complete resection, almost $50 \%$ of patients experience disease recurrence within 5 years. ${ }^{9}$ As a result, imatinib is indicated for adjuvant treatment of adult patients following resection of GIST expressing KIT (CD117), ${ }^{10-12}$ and the National Comprehensive Cancer Network (NCCN) guidelines suggest a duration of at least 36 months of imatinib in patients at high risk of recurrence. ${ }^{11}$

Targeted, oral medications are being developed increasingly for cancer treatment. In spite of the severity of many cancers, adherence to these self-administered medications can be a challenge. One study showed that patients taking oral cancer medications may overestimate their adherence to therapy by a factor of $2 .{ }^{13}$ Results from another study of patients with $\mathrm{KIT}^{+}$GIST or Philadelphia chromosome-positive chronic myeloid leukemia on imatinib therapy showed that only half of the patients achieved $100 \%$ compliance over a 24 -month period. ${ }^{14}$ Moreover, a comprehensive patient record review of patients with GIST (adjuvant: 147 records; metastatic: 225 records) who were treated with imatinib showed that less than half of the patients were perfectly compliant over their last four prescription refills (all refills exactly on time; data not published). In addition, approximately $10 \%-14 \%$ of GIST patients missed $10 \%$ or more of their prescribed daily dose during the last four refills in the study.

Effective management of adverse events (AEs) in patients with GIST can facilitate continued treatment and adherence to TKI treatment. Although imatinib is generally well tolerated, virtually all patients with metastatic GIST (>95\%) who have been treated with imatinib experienced at least one AE of any grade. ${ }^{415-18}$ However, many of the AEs occurring in imatinibtreated patients with metastatic GIST may be related to surgical procedure, underlying malignancy, or other illnesses that may occur during prolonged imatinib administration.
Significantly longer progression-free survival has been observed in patients with metastatic disease on continuous imatinib therapy compared with patients whose therapy was stopped after 1, 3, or 5 years. ${ }^{6,7,18}$ Even for patients who have achieved complete response, discontinuation can result in loss of remission and rapid tumor progression. ${ }^{6}$ Moreover, interruption of continuously scheduled treatment due to nonadherence or other reasons can lead to low imatinib plasma concentrations, which have been reported to be associated with a short median time to progression for patients with advanced GIST, confirming the importance of maintaining optimal drug levels through adherence. ${ }^{19}$ Dose escalation of imatinib may be considered in patients showing objective signs of disease progression at a lower dose and in the absence of severe adverse drug reactions. ${ }^{11}$ In cases where maximum supportive care with imatinib is not effective, or where imatinib results in life-threatening side-effects, sunitinib is recommended as second-line therapy. ${ }^{11}$

The treatment of GIST involves not only an understanding of medical therapy regimens and their associated AEs, but also an understanding of the patient emotional journey through diagnosis and treatment. The current study explored the GIST patient journey from diagnosis through the start of TKI treatment, initial response, if any, to treatment, loss of response in a subset of patients deriving clinical benefit from TKI therapy, and subsequent switching to second-line or investigational therapies. Ethnographic tools to examine patients' experience with issues such as adherence, disease knowledge, disease management, and relationship with HCPs were used.

\section{Methods \\ Study design}

Patients were recruited from individual clinical practice centers. Exclusion criteria included: patients working in advertising; marketing/market research; public relations; any media (eg, TV, radio); the pharmaceutical industry; the health care industry as physicians, pharmacists, mental health professionals, or registered/licensed nurses; and patients aged younger than 21 years or older than 80 years.

The ethnographic approach sought to engage patients with GIST in their own environments to obtain open feedback on their everyday experiences. Multiple, in-context techniques, including individual and group interviews, life histories, and participant observation, were used. Together, these methods served to elicit patients' perspectives on their disease and treatment. Researchers documented all interactions and observations with participants by taking field notes; these notes were then examined to address key topic areas (see 'Content and 
questions' section). Patient perspectives were then compared, and any emerging concepts or themes were assembled.

The main approach included a 2.5-hour, in-home interview with patients who either had resected or metastatic GIST, and their immediate family members. The patient sample comprised North American (United States and Canada) and global patients (Brazil, France, Germany, Russia, and Spain). After the interview, patients from North America, Brazil, and France completed a 7-day photo journal followed by a 45-minute telephone interview. All patients provided written consent to participate in the investigation. Patient privacy is maintained based on a signed consent agreement between the researchers and the patient participants and their families. Per the consent document, we maintain GIST patient anonymity but have permission to use patient quotes.

\section{Content and questions}

The ethnographic interview protocol included seven sections with suggested discussion time limits to provide a general structure to the interview (Table 1). These seven sections addressed issues relating to daily life, management techniques

Table I Interview protocol

\begin{tabular}{|c|c|c|}
\hline Section & Main topic & Subtopics \\
\hline I (I0 minutes) & $\begin{array}{l}\text { Personal/family } \\
\text { context }\end{array}$ & $\begin{array}{l}\text { - Background } \\
\text { - Family } \\
\text { - Employment }\end{array}$ \\
\hline 2 (45 minutes) & $\begin{array}{l}\text { Illness and } \\
\text { treatment history }\end{array}$ & $\begin{array}{l}\text { - Medical history (timeline) } \\
\text { - Diagnosis } \\
\text { - Treatment history } \\
\text { - Experience with imatinib } \\
\text { - Role imatinib plays in your life } \\
\text { - Diagnostics and switching } \\
\text { medications }\end{array}$ \\
\hline 3 (20 minutes) & $\begin{array}{l}\text { Relationship with } \\
\text { medical team }\end{array}$ & $\begin{array}{l}\text { - Physicians and facilities } \\
\text { - Provider selection process } \\
\text { - Frequency of visits } \\
\text { - Quality of relationship } \\
\text { - Source of education about } \\
\text { disease and treatments }\end{array}$ \\
\hline 4 (20 minutes) & $\begin{array}{l}\text { Current } \\
\text { management } \\
\text { of illness }\end{array}$ & $\begin{array}{l}\text { - Logistical and financial concerns } \\
\text { - Educational resources for } \\
\text { disease treatments } \\
\text { - Routines to help manage disease } \\
\text { - Lifestyle modifications }\end{array}$ \\
\hline 5 (15 minutes) & Support & $\begin{array}{l}\text { - Family support } \\
\text { - Provider support } \\
\text { - Other resources }\end{array}$ \\
\hline 6 (20 minutes) & $\begin{array}{l}\text { Impact of cancer } \\
\text { on life }\end{array}$ & - Emotional impact \\
\hline 7 (20 minutes) & $\begin{array}{l}\text { Medicine cabinet } \\
\text { tour }\end{array}$ & $\begin{array}{l}\text { - Locations of medications } \\
\text { - Medication adherence routines }\end{array}$ \\
\hline
\end{tabular}

for adherence and TKI-related side-effects, their relationship with their HCP, and how these patients perceived their disease and associated treatment options. Questions were developed by the investigators with input from specialists as well as psychosocial scientists trained in evaluating patient experiences. The interviews were audiotaped. National data were analyzed by researchers at each site, and the composite national results for the global dataset were then analyzed by two independent researchers.

\section{Results}

\section{Participants and settings}

Fifty patients with GIST were interviewed and included in the study (Table 2). A total of five patients from each country outside North America with either (a) resected primary GIST who received adjuvant imatinb therapy $(n=2)$, or (b) metastatic GIST who received TKI treatment $(n=3)$, were interviewed between February 15, 2010, and May 31, 2010. The North American country samples consisted of ten patients from the United States (adjuvant) and 15 from Canada ( $n=5$, adjuvant; $n=10$, metastatic) who were interviewed between January 19, 2010, and April 19, 2010.

\section{Overview of the stages of the GIST patient journey}

The GIST patient journey included stages of: crisis, hope, adaptation, new normal, and uncertainty (Figure 1). Patients experienced crisis when they were given the diagnosis of GIST and were faced with the ensuing treatment decisions that spark worry about the road ahead and mortality. The crisis dissipated as patients saw a therapeutic path forward, and hope emerged with drug treatment, and in those patients who had an early response, followed by disease regression or stability. Patients then entered a period of adaptation, where a prolonged period of adjustment required changes in behavior, outlook, and lifestyle. This period culminated in altered expectations and acceptance of a new outlook on life. Most patients eventually "moved on with life" in the stage of new normalcy. The new normal

Table 2 GIST treatment setting and patient nationality

\begin{tabular}{lll}
\hline Country & Adjuvant patients & Metastatic patients \\
\hline Brazil & 2 & 3 \\
Canada & 5 & 10 \\
France & 2 & 3 \\
Germany & 2 & 3 \\
Russia & 2 & 3 \\
Spain & 2 & 3 \\
United States & 10 & 0 \\
\hline
\end{tabular}

Abbreviation: GIST, gastrointestinal stromal tumors. 


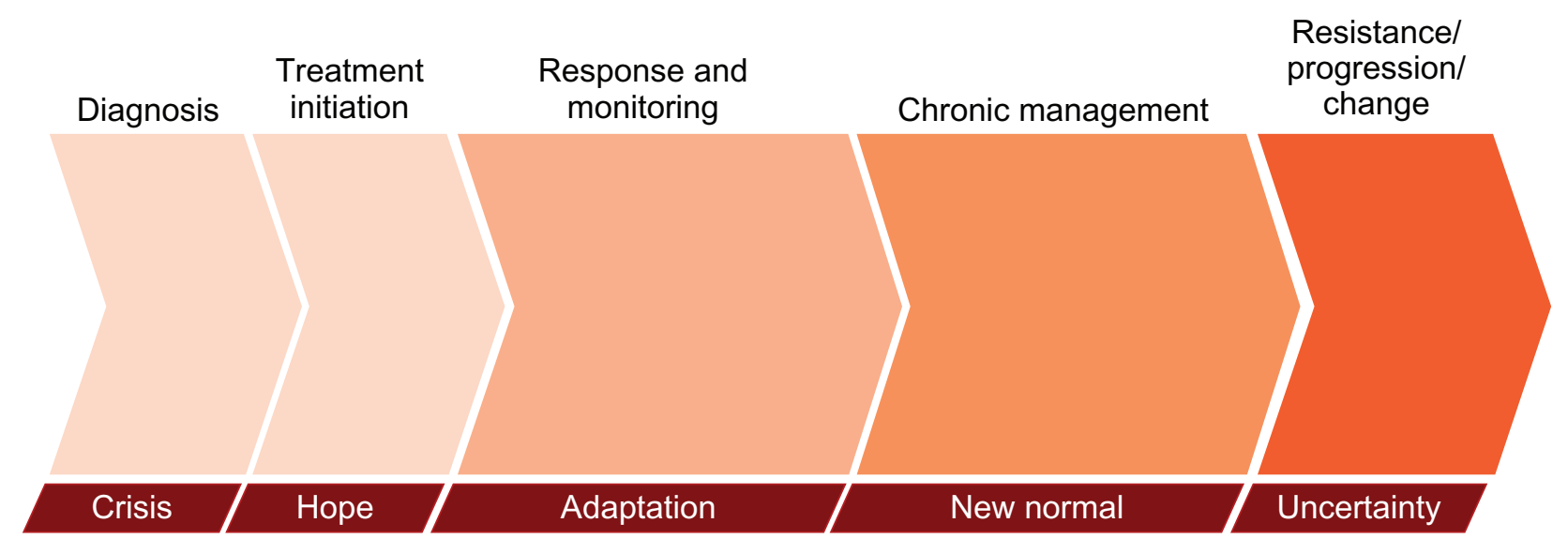

Figure I The GIST patient journey diagram. The various stages are not drawn to any scale in regard to duration, and time spent in each stage may vary between individual patients.

for these patients was markedly different, both mentally and physically, from their life before receiving a GIST diagnosis. The stage of uncertainty included fear of and/or experience of treatment failure that precipitated anxiety about the disease. Progression triggered crisis, and during this stage patients commonly searched for information on GIST and new treatment options that would bring the disease back under control. Patients progressed through these stages over time, and the stages were abbreviated or prolonged depending on the degree of each individual's disease (eg, primary resected or metastatic, comorbidities), therapy response, and circumstances.

The patient journey has changed with the availability of new clinical treatment options, information resources, and disease awareness among patients and physicians. The success of imatinib has altered how patients think about GIST; patients noted that they considered TKI treatment to be "easy" compared with how they perceived other cancer treatments are (ie, chemotherapy), and the proven outcomes of imatinib gave physicians confidence and gave patients optimism. The evolution of cancer treatment paradigms with new therapies provides imatinib-resistant or imatinib-intolerant patients with additional treatment options. These additional options along with improved outcomes meant that the uncertainty was not always a result of drug resistance or disease progression, but could also result from anxiety surrounding disease monitoring, treatment side-effects, or dose changes.

The GIST patient journey has been extended with the advent of second-line treatment with the multitargeted TKI sunitinib (Sutent $^{\circledR}$; Pfizer, New York, NY) and other investigational therapies currently in development. The NCCN guidelines recommend sunitinib $37.5 \mathrm{mg}$ once daily without interruption or sunitinib $50 \mathrm{mg}$ once daily on a schedule of 4 weeks on followed by 2 weeks off. ${ }^{11}$ When a patient can no longer tolerate, or becomes resistant to, imatinib, second-line and investigational therapies can offer hope, but these therapies can also cause patients to revisit earlier journey stages. Patients reported returning to a period of crisis with disease recurrence (adjuvant patients) or progression (metastatic patients). With the assistance of their HCPs and support network, patients may then readapt to the new stage of their journey followed by a different new normal period.

\section{Crisis}

For patients with GIST, diagnosis of the disease began the tumultuous journey of discovery about GIST and its associated prognoses, treatment options, and paradigms. Regardless of the time to the diagnosis of GIST, the stage of crisis inevitably ensued. In the case of protracted diagnoses, many patients had longstanding indeterminate symptoms, or the sense of knowing that something was "not right." Some patients went through months, even years, of anxiety, generalized symptoms, and declining health before receiving the diagnosis of GIST. One patient said, for example:

\footnotetext{
"It took months. I went to a doctor, and he didn't know what was wrong. They did one gastroscopy and colonoscopy after another until they finally did a [computed tomography scan] - then they knew it."
}

Some patients received the diagnosis quickly and incidentally, or after an acute event. One patient explained, for example:

"[In a routine procedure] they found a bump on my rectum, but I thought it was no big deal. The shock was a week later when the doctor called and said it was a very rare disease called GIST." 
Patients reported that shock is a central part of crisis, which results from diagnosis, because GIST is relatively unknown to most patients, who have never heard of this disease. The feeling of fear was heightened in patients who were told by their HCPs that they had a "rare" disease.

At diagnosis, patients learned that they had a tumor, but most patients reported that the word "cancer" was not part of the conversation. Few patients reported hearing this word at diagnosis. For example:

"The diagnosis was evasive. The radiologist said 'GIST', but at the time GIST didn't mean cancer. But [my wife] checked out the Internet and saw it was cancer."

\section{Hope}

For patients, hope came from the knowledge that there is an existing treatment with proven efficacy in GIST, so that they could see a path forward. Some patients felt psychologically reassured and physically improved when they began treatment. As one patient explained:

"They said [the therapy] might shrink the tumor. I felt better quickly, fantastic, in 2 weeks. It was an amazing difference. I learned later that shrinkage meant it would liquefy [ie, cystification] inside.”

Hope also fueled patients' search for current information about their disease and its associated treatment options. Many patients reported encountering out-of-date, contradictory, or incorrect information, which was confusing at times, as well as anxiety provoking. For example:

"I checked Google with "stromal tumors" or "GIST." Google seemed to show older results, dating back to 2002 or 2003, and that was worrisome."

Although patients uniformly grasped the idea that imatinib is a treatment and not a cure for metastatic GIST, differences in the levels of confusion arising from undefined treatment durations were observed for patients with different treatment modalities (ie, therapy in the adjuvant or metastatic and/or unresectable disease settings). Patients with metastatic GIST understood that they would most likely be on therapy for life. However, many patients who underwent surgery for primary GIST were unsure how long they would receive adjuvant treatment, because their HCPs were themselves unsure. The optimal duration of adjuvant imatinib treatment is currently unknown. As a result, treatment duration for these patients is commonly not specified by physicians upfront, and patients reported that imatinib therapy came with an indeterminate timeline they found to be unsettling. As one patient explained:

"With metastases, you will be treated with [imatinib]

automatically, but clinical data is not clear for adjuvant.

Everybody has his own opinion here."

Patients also expressed frustration at the lack of communication and coordination among the physicians responsible for their treatment. Patients reported that surgeons failed to set postsurgical expectations, while oncologists were focused on treating the cancer but provided little guidance on postsurgical lifestyle management.

\section{Adaptation}

As patients with GIST settled into medical therapy, they struggled with profound changes. Patients progressed through a period of adaptation, whereby they came to terms with the course of events: a cancer and/or rare-disease diagnosis, surgery, medications, and a harsh confrontation with their own mortality. During this transitory period, many patients reported suffering from symptoms of depression, anxiety, and insomnia. Adaptation also brought the physical challenges of postsurgery functionality, including changes in diet brought about by altered digestion, and more broad-spectrum lifestyle changes arising from increased fatigue. For example:

"Anyone who goes through a gastrectomy knows that things don't work the same anymore. I used to wonder if my symptoms were from the drug, but now I know that these things are due to my surgery. They took out a lot of parts!"

Adaptation meant an altered sense of the patient's own body. Patients expressed a myriad of feelings about having cancer in their bodies, referring to it as an "invader" or "alien." During this stage, patients with metastatic GIST adjusted to the continuous presence of cancer, while patients in the adjuvant setting adjusted to the potential risk for recurrence. Although few understood the cause of the cancer, many reported that GIST was a "wake-up call." Many became focused on the "important" things in life, such as families, relationships with others, and spirituality during this stage.

Over time, patients with GIST developed methods for managing their disease and treatment (ie, self-care). A key element of developing self-care involved coming to terms with the necessity of drug adherence, either in the adjuvant or metastatic disease setting. Most patients were motivated to take their medication, as it gave them an active role in their long-term health. Many patients acknowledged that it took time to develop steady, consistent, drug-taking routines, 
and that they needed to adapt and learn to manage treatment side-effects.

During adaptation, patients also developed a sense of personal advocacy, whereby they fostered and strengthened new relationships with their HCPs and other members of the GIST community. Having recognized that GIST is a disease that few people know about or understand, many patients felt "singled out" and were obliged to become their own best advocates. As a means of personal advocacy, patients sought out GIST specialists and often sought guidance from other patients dealing with similar experiences by participating in online communities. This personal momentum may help contribute to patients returning to a new sense of normalcy.

\section{New normal}

For patients with GIST, the new normal stage meant achieving a semblance of life pre-GIST diagnosis. New normal came as patients moved beyond merely adapting to their new circumstances, but started participating in activities within their new circumstances; for many, it was a time of returning to activities they used to do.

Of special note, all participating patients claimed that the need to adhere to TKI therapy was a prevailing thought during this stage, and most claimed that they had established routines to try to remember to take their medications. For example, many patients stored their drugs in the kitchen; some also reported filling pill boxes at a given time each week. Other strategies patients used to remain adherent included: having a family member remind them to take their medication, setting reminder mechanisms, and keeping a calendar. In addition, some patients took their medication at regularly spaced periods during the day, while others took it all at once at a specific time. Patients also viewed TKI therapy as an "insurance policy" against progression (those with metastatic and/or unresectable disease) and recurrence (those receiving adjuvant therapy). For example:

"When I walk my dog, I take a golf club, just in case the coyotes return. I consider my [imatinib] to be like a golf club, something there to protect me just in case the cancer returns."

For patients with GIST, normal life was punctuated by moments of fragility. All patients experienced increased anxiety leading up to their quarterly or biannual checkups, which included surveillance imaging scans; this increased stress is referred to, in the GIST community, as "scanxiety." In response to being informed of an "all-clear" scan (ie, no detected progression or recurrence), patients felt a palpable sense of relief, and some reported feeling they now had "time to breathe."

"When my doctor tells me that I am still cancer free, I feel so many emotions. ... I pretty much live from scan to scan."

Most patients with GIST in this study became "hypersurveillant" with their health. This attitude was evident regardless of treatment setting (metastatic or adjuvant), or time since diagnosis. Patients were sensitive to any physical changes that could signal progression (for metastatic disease) or recurrence (for resected GIST). Although patients in this study began to wonder what long-term TKI therapy may be doing to their bodies, few expressed strong concerns about potential side-effects arising from the long-term therapy. For most patients, their desire to continue treatment outweighs the potential risks.

The new normal did not mean complete resolution of all patients' emotional issues and concerns. Patients had trouble conceptualizing the details of their cancer, and perceived their disease in absolute dichotomous terms (ie, they either had GIST or were disease-free). The degree of tumor burden was irrelevant to patients, and all wanted their tumor "gone." Patients with metastatic GIST were clearly aware that their cancer was ever-present. Patients treated in the adjuvant setting worried that their current state - "no evidence of disease" - was tenuous or temporary. Many patients believed that tumor biopsies at diagnosis may have seeded cancer in their bodies, or that surgeons may not have achieved clear margins during resection. These preconceptions lead to a relative degree of uncertainty about the nature of their illness and its associated treatments.

\section{Uncertainty}

Patients experienced periodic episodes of uncertainty for various reasons along their disease journey and at various times. Clinical changes, uncertain test results, and new disease symptoms or treatment side-effects triggered uncertainty, whereby patients regressed to previous emotional patterns. Patients in both the adjuvant and metastatic settings in this study tended to cycle back through all the stages (crisis, hope, adaptation, new normal) during episodes of uncertainty; adjuvant patients responded to potential events or symptoms with emotions similar to metastatic patients, despite their lack of evidence of disease.

Many patients with GIST felt the need to stay abreast of new information. However, new scientific data, especially for adjuvant therapy, could trigger uncertainty. For example, reading new GIST studies or new clinical trial data led to 
anxiety as patients interpreted or misinterpreted results. Patients on adjuvant therapy carefully followed changing information about duration of treatment and were continuously concerned with whether stopping adjuvant therapy would allow for disease recurrence.

Switching therapy due to TKI resistance, disease recurrence, or progression was a key trigger for uncertainty and often precipitated a step back in the emotional journey (Figure 2). Patients believed that this meant that the worst outcome had occurred, and many patients reverted to crisis. Most patients were devastated by TKI failure and their unknown future path. Switching therapies also revived information-seeking behavior as patients re-energized around the need for personal advocacy. For example, one patient questioned:

"What will happen now? Will this new drug work as well as [the last drug]?"

Switching drugs due to intolerance often brought relief instead of crisis. Metastatic or adjuvant patients who suffered intolerance to a TKI often readapted to the new therapy with relative ease, and their physical and emotional outlooks improved considerably after switching. Eventually these patients settled back to the normalcy of tolerable side-effects and good response. Conversely, emergence of new sideeffects after switching therapy could lead affected patients to experience $c r i s i s$, as one patient explained:

"When I went on the [new] med, it was really tough. I had a hard time on it, because of the side-effects. Compared to what I am enduring now, [the prior therapy] was a cakewalk."

\section{Discussion}

This global ethnographic study explored the GIST patient journey from diagnosis, through initiation of treatment, response to treatment in most patients, loss of response, and switching to second-line or investigational therapies. The study revealed that patients with GIST progressed through periods of crisis, hope, adaptation, new normal, and uncertainty.

For patients with metastatic GIST, persistent uncertainty represented the overriding reality (Figure 3 ). The impact of the disease was very high, and each stage of the journey was difficult. Crisis involved making high-stakes decisions within a vacuum of knowledge and misunderstanding. Hope for treatment success was tempered by the reality of a limited prognosis. Adaptation was prolonged and painful, and coming to terms with the presence of cancer was daunting. The new normal was a fragile time - physically sustainable but emotionally tumultuous. Uncertainty was a frequent occurrence even with stable disease, coinciding with anxiety experienced in anticipation of HCP visits and scans.

The adjuvant GIST patient journey displayed many similarities to the journey of the metastatic patient (Figure 4). However, the adjuvant patient experienced hope in treatment success tempered by ongoing or recent medical intervention (eg, surgery). The adaptation stage for these patients was also difficult. Shortly after resections and despite no evidence of disease, physical changes arising from surgery were profound, and emotions were raw. Patients' new normal was also imperfect, as they were hypervigilant about physical changes associated with surgery and treatment. In addition, patients with GIST in both the adjuvant and metastatic settings experienced

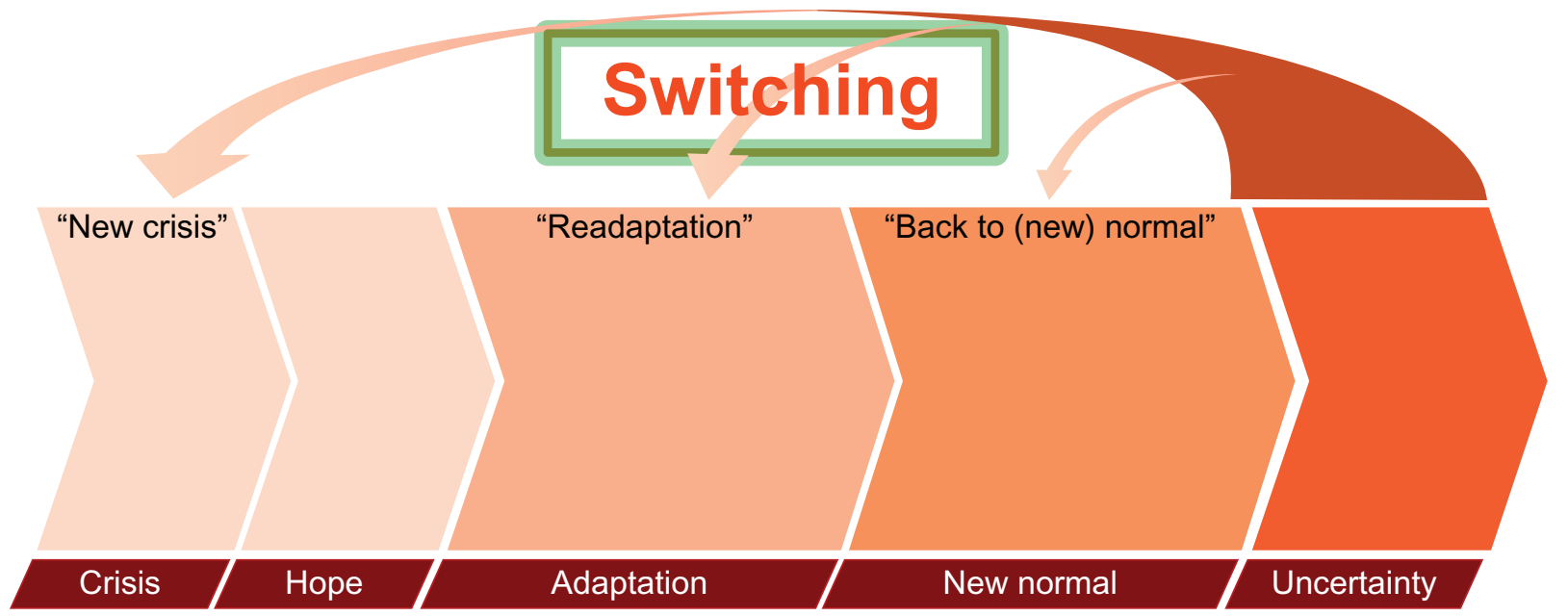

Figure 2 Switching therapy can lead to uncertainty and often precipitates a step back in the emotional journey. The various stages are not drawn to any scale in regard to duration, and time spent in each stage may vary between individual patients. 


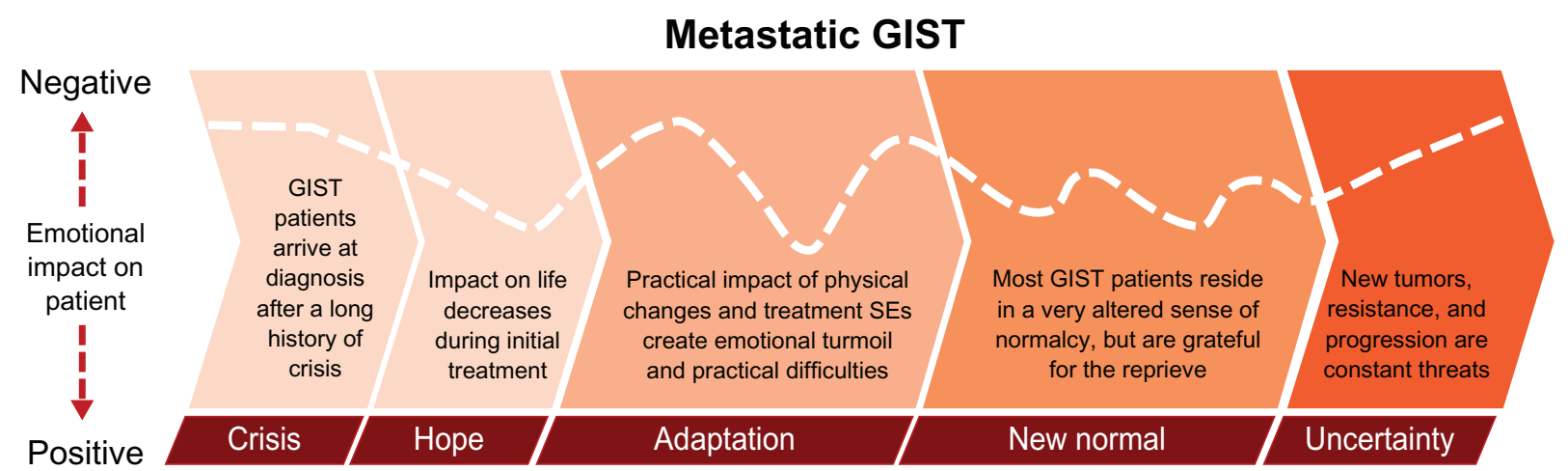

Figure 3 The metastatic GIST patient journey. The various stages are not drawn to any scale in regard to duration, and time spent in each stage may vary between individual patients.

Abbreviations: GIST, gastrointestinal stromal tumors; SEs, side-effects.

"scanxiety" prior to follow-up examinations (ie, uncertainty about the constant threat of disease recurrence/progression that heightened during their follow-up periods).

The patients with GIST in this study were highly selfdirected, but they encountered challenges finding accurate, relevant, and available information about GIST. Although a strong sense of personal advocacy drove the search for information, what patients learned was not always accurate, or they misinterpreted results from clinical trials. Thus, each interaction between a patient and an HCP offers a critical opportunity to clarify and reinforce accurate and relevant information. Physicians also could reinforce and support their search and ensure that the information patients receive is appropriate by directing them to medically accurate GIST support materials and communities. The provision of accurate information may lead to a greater sense of independence and fewer touch points with HCPs. For patients transitioning from crisis to hope, HCPs can help set expectations around drug efficacy and differentiate among treatments. HCPs can also help patients understand the real risk of disease progression and recurrence by educating them about the effectiveness of TKI therapy and providing a roadmap to those with GIST in the adjuvant setting to help them understand issues surrounding duration of treatment. Additionally, to reduce the risk of relapse or recurrence, the importance of adherence to therapy should be emphasized to patients.

As patients moved through adaptation to new normal, physicians had the chance to remind and reassure patients of the safety of drug and disease monitoring. Patients with either metastatic or primary GIST were hypervigilant about physical changes. Regular checkups during this stage offered physicians the opportunity to remind patients of the need for adherence to therapy, to reinforce to patients the favorable tolerability of TKIs, and to ensure the best possible longterm care. Most patients with GIST undergo surgery at some point in their patient journey, whether to remove the primary tumor or the metastases. Coordination and consistent communication are important between the different disciplines

\section{Adjuvant GIST}

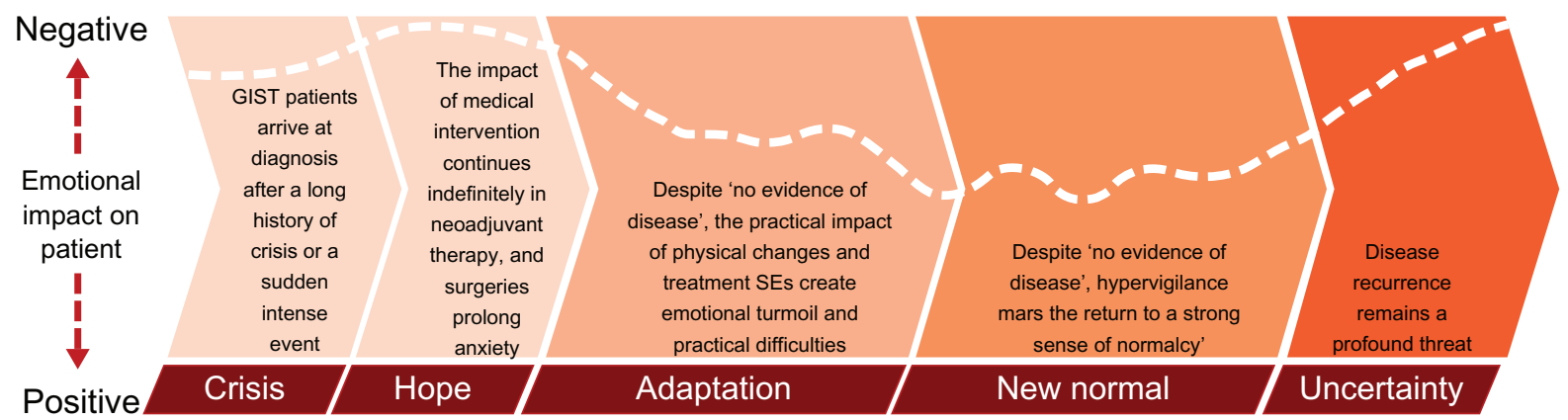

Figure 4 The adjuvant GIST patient journey. The various stages are not drawn to any scale in regard to duration, and time spent in each stage may vary between individual patients.

Abbreviation: GIST, gastrointestinal stromal tumors. 
(ie, oncologists and surgeons), as well as with the patients. This also presents an opportunity for physicians to help patients make distinctions between side-effects associated with TKI therapy and those occurring for other reasons (eg, invasive surgical procedures, illnesses unrelated to GIST) so patients do not mistakenly attribute discomfort or side-effects to their medicine with potentially consequent-reduced adherence.

\section{Limitations}

Inclusion in this voluntary study involved patients who were actively engaged and aware of their disease, and many patients participating in this study were driven to gather information; this may reflect a potential selection bias. Multiple data collection techniques were used in this study to lessen these effects. Furthermore, it is important to note that the degree of personal advocacy demonstrated by interviewed patients in this study may not be representative of the GIST patient population as a whole. Nonetheless, the general GIST patient population may require more $\mathrm{HCP}$ and staff guidance than the patients participating in this study. Although care was taken to accommodate cultural and health care system differences across the participating countries, these factors may also influence patient perceptions.

\section{Conclusion}

The GIST patient journey is evolving as more information becomes available and patients become further educated about the disease. Most patients with GIST are highly selfdirected and understand the importance of adherence to TKI therapy, while acknowledging that therapy may have an impact on their daily lives. HCPs can optimize adherence by managing patient expectations about therapy duration and dissociating medication side-effects from postsurgical events where appropriate. Each phase of the patient journey offers opportunities for physicians and staff, and drug manufacturers to provide support to patients.

\section{Acknowledgment}

The authors acknowledge Robert Gillespie, PhD, of Chameleon Communications International, who provided medical writing services with funding from Novartis Pharmaceuticals.

\section{Disclosure}

This study was sponsored by Novartis Pharmaceuticals and conducted by N Macdonald and A Shapiro while employed by Hall \& Partners Group. C Bender, M Paolantonio, and
J Coombs are shareholders and employees of Novartis Pharmaceuticals.

\section{References}

1. Perez EA, Livingstone AS, Franceschi D, et al. Current incidence and outcomes of gastrointestinal mesenchymal tumors including gastrointestinal stromal tumors. J Am Coll Surg. 2006;202(4):623-629.

2. Kindblom L-G, Meis-Kindblom J, Bumming P, et al. Incidence, prevalence, phenotype and biologic spectrum of gastrointestinal stromal cell tumors (GIST) - a population-based study of 600 cases. Annals of Oncology Abstract Book of the 27th ESMO Congress; October 18-22, 2002; Nice, France. Ann Oncol. 2002;13(Suppl 5):157.

3. Miettinen M, Lasota J. Gastrointestinal stromal tumors - definition, clinical, histological, immunohistochemical, and molecular genetic features and differential diagnosis. Virchows Arch. 2001;438(1):1-12.

4. Blanke CD, Rankin C, Demetri GD, et al. Phase III randomized, intergroup trial assessing imatinib mesylate at two dose levels in patients with unresectable or metastatic gastrointestinal stromal tumors expressing the kit receptor tyrosine kinase: S0033. J Clin Oncol. 2008;26(4):626-632.

5. Verweij J, Casali PG, Zalcberg J, et al. Progression-free survival in gastrointestinal stromal tumours with high-dose imatinib: randomised trial. Lancet. 2004;364(9440):1127-1134.

6. Le Cesne A, Ray-Coquard I, Bui BN, et al. Discontinuation of imatinib in patients with advanced gastrointestinal stromal tumours after 3 years of treatment: an open-label multicentre randomised phase 3 trial. Lancet Oncol. 2010;11(10):942-949.

7. Ray-Coquard IL, Bin Bui N, Adenis A, et al. Risk of relapse with imatinib (IM) discontinuation at 5 years in advanced GIST patients: results of the prospective BFR14 randomized phase III study comparing interruption versus continuation of IM at 5 years of treatment: A French Sarcoma Group Study. J Clin Oncol (Meeting Abstracts). 2010;28(15 Suppl):10032.

8. Joensuu H, Eriksson M, Hatrmann J, et al. Twelve versus 36 months of adjuvant imatinib (IM) as treatment of operable GIST with a high risk of recurrence: Final results of a randomized trial (SSGXVIII/AIO). J Clin Oncol. 2011;29(Suppl):LBA1.

9. DeMatteo RP, Lewis JJ, Leung D, Mudan SS, Woodruff JM, Brennan MF. Two hundred gastrointestinal stromal tumors: recurrence patterns and prognostic factors for survival. Ann Surg. 2000;231(1):51-58.

10. Gleevec/Glivec prescribing information. East Hanover: Novartis Pharmaceuticals Corporation; c2010 [revised 2010 Feb]. http://www. pharma.us.novartis.com/product/pi/pdf/gleevec_tabs.pdf. Accessed January 27, 2011.

11. Clinical practice guidelines in oncology. Soft tissue sarcoma. V.2.2011. Fort Washington: National Comprehensive Cancer Network; c2011. Available at: http://www.nccn.org/professionals/physician_gls/f_guidelines.asp. Accessed September 10, 2011.

12. Casali PG, Blay JY. Gastrointestinal stromal tumours: ESMO Clinical Practice Guidelines for diagnosis, treatment and follow-up. Ann Oncol. 2010;21(Suppl 5):v98-v102.

13. Partridge AH, Avorn J, Wang PS, Winer EP. Adherence to therapy with oral antineoplastic agents. J Natl Cancer Inst. 2002;94(9):652-661.

14. Tsang J, Rudychev I, Pescatore SL. Prescription compliance and persistency in chronic myelogenous leukemia (CML) and gastrointestinal stromal tumor (GIST) patients (pts) on imatinib (IM). J Clin Oncol (Meeting Abstracts). 2006;24(18 Suppl):6119.

15. Blanke CD, Demetri GD, von Mehren M, et al. Long-term results from a randomized phase II trial of standard- versus higher-dose imatinib mesylate for patients with unresectable or metastatic gastrointestinal stromal tumors expressing KIT. J Clin Oncol. 2008;26(4):620-625.

16. DeMatteo RP, Ballman KV, Antonescu CR, et al. Adjuvant imatinib mesylate after resection of localised, primary gastrointestinal stromal tumour: a randomised, double-blind, placebo-controlled trial. Lancet. 2009;373(9669):1097-1104. 
17. Demetri GD, von Mehren M, Blanke CD, et al. Efficacy and safety of imatinib mesylate in advanced gastrointestinal stromal tumors. $N$ Engl J Med. 2002;347(7):472-480.

18. Blay JY, Le Cesne A, Ray-Coquard I, et al. Prospective multicentric randomized phase III study of imatinib in patients with advanced gastrointestinal stromal tumors comparing interruption versus continuation of treatment beyond 1 year: the French Sarcoma Group. J Clin Oncol. 2007;25(9):1107-1113.
19. Demetri GD, Wang Y, Wehrle E, et al. Imatinib plasma levels are correlated with clinical benefit in patients with unresectable/ metastatic gastrointestinal stromal tumors. J Clin Oncol. 2009; 27(19):3141-3147.

\section{Publish your work in this journal}

Patient Preference and Adherence is an international, peer-reviewed, open access journal focusing on the growing importance of patient preference and adherence throughout the therapeutic continuum. Patient satisfaction, acceptability, quality of life, compliance, persistence and their role in developing new therapeutic modalities and compounds to optimize clinical outcomes for existing disease states are major areas of interest. This journal has been accepted for indexing on PubMed Central. The manuscript management system is completely online and includes a very quick and fair peer-review system. Visit http://www.dovepress.com/ testimonials.php to read real quotes from published authors.

Submit your manuscript here: http://www.dovepress.com/patient-preference-and-adherence-journal 